\title{
IncRNA HCP5 acts as a ceRNA to regulate EZH2 by sponging miR-138-5p in cutaneous squamous cell carcinoma
}

\author{
SHIBO ZOU, YA GAO and SHUTANG ZHANG \\ Department of Burn and Reconstruction Surgery, The First Affiliated Hospital of \\ Zhengzhou University, Zhengzhou, Henan 450052, P.R. China
}

Received June 16, 2020; Accepted January 26, 2021

DOI: $10.3892 /$ ijo.2021.5236

\begin{abstract}
Long non-coding RNAs (lncRNAs) and microRNAs (miRNAs) are essential for the progression of tumors, including cutaneous squamous cell carcinoma (CSCC). The present study aimed to examine the competing endogenous RNA (ceRNA) network in CSCC. Differentially expressed genes in CSCC were analyzed using the GSE66359 microarray data set, and the upstream miRNAs and lncRNAs were predicted using online database analysis (TargetScan 7.1, mirDIP 4.1, miRSearch V3.0, miRDB and RNA22 2.0) and were verified in clinical tissues. RNA pull-down and dual luciferase reporter gene assays were used to verify the targeting relationships among lncRNA human histocompatibility leukocyte antigen complex P5 (HCP5), miR-138-5p and enhancer of zeste homolog 2 (EZH2). Cell lines with a high and low HCP5 expression were screened, and a pcDNA-3.1-HCP5 overexpression vector, small interfering RNA against HCP5, miR-138-5p mimics and miR-138-5p inhibitors were transfected into the CSCC cells. Cell viability, invasion, migration, apoptotic rate and autophagy were evaluated. The effects of HCP5 on autophagy and apoptosis of CSCC cells were verified in vivo using Ki67 and TUNEL staining. EZH2 was demonstrated to be upregulated in CSCC cells. miR-138-5p target sequences were identified in HCP5 and EZH2. HCP5 was revealed to function as a putative ceRNA of miR-138-5p to positively regulate $\mathrm{EZH} 2$, and $\mathrm{EZH} 2$ was shown to regulate autophagy and apoptosis of CSCC cells through the STAT3/VEGFR2 pathway. HCP5 overexpression decreased miR-138-5p levels, increased EZH2 levels and promoted cell malignant behaviors and autophagy but decreased the apoptosis rate. These trends
\end{abstract}

Correspondence to: Dr Shibo Zou, Department of Burn and Reconstruction Surgery, The First Affiliated Hospital of Zhengzhou University, 1 Jianshe East Road, Erqi, Zhengzhou, Henan 450052, P.R. China

E-mail: shibozou@163.com

Key words: cutaneous squamous cell carcinoma, long non-coding RNA human histocompatibility leukocyte antigen complex P5, microRNA-138-5p, enhancer of zeste homolog 2, autophagy, STAT3/VEGFR2 pathway were opposite when HCP5 was silenced. In conclusion, HCP5 may competitively bind to miR-138-5p to regulate EZH2 in CSCC cells, promoting autophagy and reducing apoptosis through the STAT3/VEGFR2 pathway. This study may provide a new perspective for understanding the molecular mechanism and treatment of CSCC.

\section{Introduction}

Cutaneous squamous cell carcinoma (CSCC) is a malignant hyperplasia of the skin epithelium, accounting for $20-50 \%$ of all skin cancers (1). In addition to ultraviolet radiation, other CSCC risk factors include chronic immunosuppression, chronic skin disease, genetic disease, ionizing radiation, and long-term arsenic exposure (2). Patients afflicted with CSCC display a large number of precursor lesions of actinic keratosis, which typically present as papules, plaques or sclerotic nodules, with smooth, scaly, verrucous or ulcerative surfaces (2). Although most CSCCs show a relatively mild course, depending on the location, size, invasion degree, tissue differentiation, perineural invasion, and immune deficiency of tumors, they may have a local invasion and metastatic potential (3). A recent meta-analysis has suggested that risk factors include a diameter $>20 \mathrm{~mm}$, an ear or lip tumor location, invasion beyond the subcutaneous fat and the presence of perineural invasion, which have statistically significant associations with disease-specific CSCC death (4). In view of the histological grade of histopathological high-risk features, a recent study has shown that the 5-year metastasis-free and overall survival rates are noticeably higher in well-differentiated tumors $(\sim 70 \%)$ compared with moderately differentiated $(\sim 51 \%)$ and poorly differentiated ( $26 \%)$ tumors (3). Once the cancer is established, autophagy helps tumor development and allows tumor cells to survive under starvation or hypoxia (5). Autophagy inhibition has been identified as an effective therapy for CSCC treatment (6). In view of these previous reports, the present study aimed to identify new cell therapies for CSCC based on autophagy inhibition.

Long non-coding RNAs (lncRNAs) are important regulators of various biological processes, including cancer stemness and tumorigenesis (7), and their roles in CSCC cell behavior have also been widely investigated (8). LncRNA human histocompatibility leukocyte antigen complex P5 (HCP5) has been implicated in adaptive and innate immune systems and has 
been correlated with the development of various autoimmune diseases and cancers, as reviewed by Kulski (9). In addition, lncRNAs can act as competitive endogenous RNAs (ceRNAs) to sponge microRNAs (miRNAs/miRs) by competing with mRNA to bind to miRNA, thus regulating miRNA and mRNA expression patterns, and finally affecting tumor processes (10). HCP5 expression is positively associated with the pathological grade of gliomas, and HCP5 knockout exerts an antitumoral effect on gliomas through the HCP5/miR-139/Runt-related transcription factor 1 feedback loop (11). Additionally, HCP5 levels are decreased in skin cutaneous melanoma tissues and are associated with an undesirable overall survival and progression (12). Enhancer of zeste homolog 2 (EZH2) serves crucial roles in a range of biological processes, including organ development and homeostasis, gene repression and DNA damage repair (13). EZH2 is a polycomb group protein that is involved in the progression of a number of human cancers, including CSCC (14). EZH2 regulates cancer cell autophagy $(15,16)$, thus, it was also a focus of the present study. However, there is little research on the mechanism of HCP5 and EZH2 in CSCC progression; therefore, the present study aimed to discern a ceRNA network involving HCP5 in CSCC cells.

\section{Materials and methods}

Microarray analysis. Using the Gene Expression Omnibus (GEO) database (https://www.ncbi.nlm.nih.gov/geo), five healthy and eight tumor samples were obtained from a CSCC microarray (GSE66359); the healthy samples were used as the control. The limma package in $\mathrm{R}$ was used to screen the differentially expressed genes using a $\mid \log \mathrm{FCl}>1$ and a $\mathrm{P}<0.05$ as the screening standards. The upstream miRNAs of EZH2 were predicted using the TargetScan 7.1 (http://www. targetscan.org/vert_71), mirDIP 4.1 (http://ophid.utoronto. ca/mirDIP/index.jsp\#r), miRSearch V3.0 (https://www.exiqon. com/miRSearch), and miRDB (http://mirdb.org) databases. The upstream lncRNAs of miR-138-5p were predicted using the RNA22 2.0 database (https://cm.jefferson.edu/rna22).

Tissue collection. Between October 2016 and October 2018, cancer tissues and healthy skin tissues were collected from 60 patients with CSCC (33 male; 27 female; age, $53.6 \pm 8.1$ years; body mass index, 22.61 \pm 1.08$)$ admitted to The First Affiliated Hospital of Zhengzhou University (Zhengzhou, China). Patients were excluded if they had incomplete clinical data, mental or consciousness disorders, other primary malignant tumors, autoimmune diseases, serious organic diseases, important organ dysfunction or coagulation dysfunction, if they were pregnant or lactating women, and if they had an allergic constitution or related contraindications.

Cell grouping and transfections. CSCC cell lines (A431, COLO-16, SCC13, SCL-1, HSC-1, and HSC-5) and the human immortalized keratinocyte HaCaT cell line (all purchased from American Type Culture Collection) were cultured in DMEM supplemented with $10 \%$ FBS, $100 \mathrm{U} / \mathrm{ml}$ penicillin, and $100 \mathrm{U} / \mathrm{ml}$ streptomycin at $37^{\circ} \mathrm{C}$ and $5 \% \mathrm{CO}_{2}$.

EZH2 cDNA and IncRNA HCP5 were cloned into pcDNA3.1 (Invitrogen; Thermo Fisher Scientific, Inc.) to construct the overexpression vectors. miR-138-5p mimics, mimics negative control (NC), miR-138-5p inhibitor, inhibitor NC (inhi-NC), small interfering RNA (si)-HCP5-1, si-HCP5-2 and si-NC were designed and synthesized by Shanghai GenePharma Co., Ltd. The details are provided in Table I.

si-HCP5-1, si-HCP5-2, si-NC, miR-138-5p mimics and mimics-NC, miR-138-5p inhibitor and inhi-NC were transfected into A431 cells. pcDNA3.1-NC and pcDNA3.1-HCP5 were transfected into SCL-1 cells. miR-138-5p mimics and mimics-NC were transfected into SCL-1 cells and SCL-1 cells overexpressing HCP5. pcDNA3.1-NC and pcDNA3.1-EZH2 were co-transfected into SCL-1 cells and A431 cells with si-HCP-1 and si-HCP5-2; SCL-1 cells transfected with pcDNA3.1-EZH2 were pre-treated with STAT3/VEGFR2 pathway inhibitor AG-490 (10 nM; AmyJet Scientific Co., Ltd.) for $24 \mathrm{~h}$ at $37^{\circ} \mathrm{C}(17)$. Briefly, cells to be transfected were seeded in 6 -well plates $\left(1.0 \times 10^{5}\right.$ cells/well $)$ and grown overnight for $18 \mathrm{~h}$. Cells at $80 \%$ confluence were transfected with 100 pmol siRNAs, miRNA mimics, miRNA inhibitors or the respective controls using Lipofectamine ${ }^{\circledR} 2000$ (Invitrogen; Thermo Fisher Scientific, Inc.) following the manufacturer's instructions. After $48 \mathrm{~h}$ of incubation at $37^{\circ} \mathrm{C}$, cells were collected for subsequent experiments.

Reverse transcription-quantitative polymerase chain reaction $(R T-q P C R)$. Total RNA was obtained by the one-step method using TRIzol ${ }^{\circledR}$ (Invitrogen; Thermo Fisher Scientific, Inc.), and high-quality RNA was confirmed using ultraviolet analysis (A260/280) and formaldehyde denaturation electrophoresis. qPCR was performed following the manufacturer's instructions of TaqMan ${ }^{\text {TM }}$ Sample-to-SNP ${ }^{\text {TM }}$ kit (cat. no. 4403313; Applied Biosystems; Thermo Fisher Scientific, Inc.). PCR primers were designed and synthesized by Sangon Biotech Co., Ltd. and are listed in Table II. GAPDH was used as the internal reference for IncRNAs and U6 was used as the internal reference for miRNAs; expression data were normalized to GAPH or U6. Amplification and dissolution curves were confirmed after the reaction. The relative expression was calculated using the $2^{-\Delta \Delta \mathrm{Cq}}$ method (18).

Western blot analysis. Cells were lysed for $30 \mathrm{~min}$ using a cold radio-immunoprecipitation assay buffer containing a protease inhibitor mixture (Sigma-Aldrich, Merck KGaA). Lysates were centrifuged at $4^{\circ} \mathrm{C}$ and $16,000 \mathrm{x}$ g for $20 \mathrm{~min}$, and the supernatant was obtained. Protein concentration was determined using the bicinchoninic acid kit (Beyotime Biotechnology Co., Ltd.). Proteins were separated by $10 \%$ SDS-PAGE and transferred onto polyvinylidene fluoride membranes. The membranes were blocked with 5\% skim milk for $2 \mathrm{~h}$ at room temperature. After incubation with the primary antibodies (Table III) overnight at $4^{\circ} \mathrm{C}$, the membranes were incubated with goat anti-rabbit $\mathrm{IgG}$ (1:2,000; cat. no. ab205718; Abcam) or goat anti-mouse IgG (1:2,000; cat. no. ab205719; Abcam) secondary antibody for $1 \mathrm{~h}$ at room temperature, were developed using the Immobilon Western Chemiluminescent HRP substrate (cat. no. WBKLS0100; MilliporeSigma), and visualized using an imager (Bio-Rad, Inc.). Protein expression levels were semi-quantified using ImageJ Pro Plus 6.0 [National Institutes of Health (NIH)]. 
Table I. siRNAs and miRNA mimics sequences.

\begin{tabular}{ll}
\hline Name & \multicolumn{1}{c}{ Sequence $\left(5^{\prime} \rightarrow 3^{\prime}\right)$} \\
\hline si-HCP5-1 & GGCTCAACTCACAAGAAAC \\
si-HCP5-2 & TTCTCCGAACGTGTCACGT \\
si-NC & TTCTCCGAACGTGTCA CGT \\
miR-138-5p mimics & AGCUGGUGUUGUGAAUCAGGCCG \\
mimics-NC & CAGUACUUUUGUGUAGUACAA \\
miR-138-5p inhi & CGGCCUGAUUCACAACACCAGCU \\
inhi-NC & CAGUACUUUUGUGUAGUACAA \\
\hline
\end{tabular}

HCP5, histocompatibility leukocyte antigen complex P5; inhi, inhibitor; miR, microRNA; NC, negative control; si, short hairpin RNA.

Table II. Primer sequences used in reverse transcription-quantitative PCR.

\begin{tabular}{|c|c|}
\hline Gene & Primer sequence $\left(5^{\prime} \rightarrow 3^{\prime}\right)$ \\
\hline \multirow[t]{2}{*}{$\mathrm{HCP} 5$} & F: GACTCAGATTCTCCCCAGACGC \\
\hline & R: GTGGGATCCACAACACTTTAAT \\
\hline \multirow[t]{2}{*}{$G A P D H$} & F: GGGAGCCAAAAGGGTCAT \\
\hline & R: GAGTCCTTCCACGATACCAA \\
\hline \multirow{2}{*}{ HAGLROS } & F: GCTCTCCGCGCCTCAGCGAGCGG \\
\hline & R: ACAGCAGTCTTAGCCTACTTCCT \\
\hline \multirow[t]{2}{*}{$E Z H 2$} & F: ATGGGCCAGACTGGGAAGAAA \\
\hline & R: GGAGGTAGCAGATGTCAAGGG \\
\hline \multirow[t]{2}{*}{ LINC00839 } & F: GATATCTAGTTAAATAAGTCAT \\
\hline & R: GGGAAATCAGGGGTCTCACAGC \\
\hline \multirow[t]{2}{*}{$P S M B 8-A S 1$} & F: TCCACATCCCCCTGCCTTTTCCGAG \\
\hline & R: CAGACAGCGTCCCGCTATGTTGCC \\
\hline \multirow[t]{2}{*}{ PP7080 } & F: GACGCCGCCGGCGGGAAGGGTCC \\
\hline & R: CCCTGTCTGGACACTGCCAACCC \\
\hline \multirow[t]{2}{*}{ LINC01139 } & F: TGGAATTCAAGCTGTGGGTGAGAA \\
\hline & R: ATACGGCCAGAGCGAGTCTACCCT \\
\hline \multirow[t]{2}{*}{ LINC00963 } & F: CCGGCCCGTCTCGGGGCCCTGAGTC \\
\hline & R: GTGAGCCCCAGCCAAGATGAAGGC \\
\hline \multirow[t]{2}{*}{ FLVCRI-AS1 } & F: GAGAACTTCGGGAGGCGGGGGAGG \\
\hline & R: GATCTCAAGACGGTGTCTGGCACAT \\
\hline \multirow[t]{2}{*}{ U6 } & F: CGCTTCGGCAGCACATATAC \\
\hline & R: AATATGGAACGCTTCACGA \\
\hline \multirow[t]{2}{*}{ miR-138-5p } & F: AGCTGGTGTTGTGAATCAGG \\
\hline & R: CGGCCTGATTCACAACACCA \\
\hline \multirow[t]{2}{*}{$\operatorname{miR}-26 a-5 p$} & F: TTCAAGTAATCCAGGATAGGCT \\
\hline & R: AGCCTATCCTGGATTACTTGAA \\
\hline \multirow[t]{2}{*}{ miR-26b-5p } & F: TTCAAGTAATTCAGGATAGGT \\
\hline & R: ACCTATCCTGAATTACTTGAA \\
\hline \multirow[t]{2}{*}{ miR-1297 } & F: TGTTTATCTCTAGGGTTGATC \\
\hline & R: GTTTCACTACACCTGAATTAC \\
\hline \multirow[t]{2}{*}{$\operatorname{miR}-4465$} & F: CATGTGTCCCCTGGCACGCTA \\
\hline & R: TCATGTGTCCCCTGGTCAGACT \\
\hline
\end{tabular}

AS, antisense RNA; EZH2, enhancer of zeste homolog 2; F, forward; HCP5, histocompatibility leukocyte antigen complex P5; miR, microRNA; R, reverse; PCR, polymerase chain reaction.
Table III. Antibodies used for western blot analysis.

\begin{tabular}{lcc}
\hline Antibody & Catalog number & Dilution ratio \\
\hline$\beta$-actin & ab8227 & $1: 1,000$ \\
LC3B & ab48394 & $1: 1,000$ \\
p62 & ab56416 & $1: 1,000$ \\
Bcl-2 & ab182858 & $1: 2,000$ \\
Bax & ab32503 & $1: 1,000$ \\
EZH2 & ab186006 & $1: 1,000$ \\
p-STAT3 & ab76315 & $1: 2,000$ \\
STAT3 & ab119352 & $1: 5,000$ \\
VEGFR2 & ab11939 & $1: 1,000$
\end{tabular}

Bax, Bcl-2-associated X; Bcl-2, B-cell lymphoma-2; EZH2, enhancer of zeste homologue 2 ; LC3B, light chain $3 \mathrm{~B}$; p-, phosphorylated; STAT, signal transducer and activator of transcription; VEGFR2, vascular endothelial growth factor receptor 2 .

Cell Counting Kit-8 (CCK-8) assay. The CCK-8 assay was used to determine cell viability following the manufacturer's instructions (cat. no. C0038; Beyotime Institute of Biotechnology). CSCC cells with different transfections were seeded onto 96-well plates at a density of 5,000 cells/well. After incubation at $37^{\circ} \mathrm{C}$ for $2 \mathrm{~h}$, the media was replaced with $100 \mu \mathrm{l}$ CCK-8 solution (90 $\mu \mathrm{l}$ serum-free DMEM and $10 \mu \mathrm{l}$ CCK-8) at $0,12,24,36$ and $48 \mathrm{~h}$ incubation. The absorbance at $450 \mathrm{~nm}$ was then measured for each well.

Transwell assay and scratch test. A 24-well Transwell system (8 $\mu \mathrm{m}$ pore size membrane; Corning, Inc.) and a filter membrane pre-coated with Matrigel (BD Biosciences) were used to evaluate cell invasion. Suspensions of the transfected CSCC cells $\left(1 \times 10^{5}\right.$ cells $\left./ \mathrm{ml}\right)$ were prepared in serum-free DMEM $(0.1 \%$ bovine serum albumin) and placed onto the apical chamber of the Transwell insert; the basolateral chamber was filled with DMEM containing 10\% FBS. The Transwell chamber was incubated for $24 \mathrm{~h}$ at $37^{\circ} \mathrm{C}$ and $5 \% \mathrm{CO}_{2}$. Subsequently, the filter membrane was removed and rinsed with PBS, fixed with $0.5 \%$ glutaraldehyde for $30 \mathrm{~min}$ at room temperature and stained with crystal violet for $30 \mathrm{~min}$ at $37^{\circ} \mathrm{C}$ and $5 \% \mathrm{CO}_{2}$. Five random fields of vision were selected for cell counting under a light microscope (magnification, x200).

Transfected CSCC cells were detached and collected using $0.25 \%$ trypsin, and then resuspended to $2 \times 10^{5}$ cells $/ \mathrm{ml}$ in DMEM containing $10 \%$ FBS. The cells were seeded onto 6-well plates $\left(2 \mathrm{ml} /\right.$ well) and cultured at $37^{\circ} \mathrm{C}$ and $5 \% \mathrm{CO}_{2}$ for $12 \mathrm{~h}$. Following cell adherence, a straight line perpendicular to the center was scratched on the cell monolayer using a pipette tip. Subsequently, the floating cells were washed away using PBS, and cells were continuously cultured for $12 \mathrm{~h}$. Images were captured under a phase contrast microscope at 0 and $12 \mathrm{~h}$ incubation. ImageJ Pro Plus $6.0(\mathrm{NIH})$ was utilized to analyze the scratch width of cells in different groups and time periods. Cell migration $=$ scratch width at $0 \mathrm{~h}$-scratch width at $12 \mathrm{~h}$.

Flow cytometry. When the transfected cells were cultured to $85 \%$ confluence, the suspended cells in the culture supernatant 
were collected into the centrifuge tube. The adherent cells were detached with $0.25 \%$ trypsin, washed three times with PBS, and collected into the centrifuge tube in the previous step. Next, the cells were centrifuged at $4^{\circ} \mathrm{C}$ and $150 \mathrm{x}$ g for $5 \mathrm{~min}$. The cells were resuspended with cold PBS and centrifuged for $5 \mathrm{~min}$ at $150 \mathrm{x} \mathrm{g}$ at $4^{\circ} \mathrm{C}$ to collect cell precipitates. This procedure was repeated twice to remove residual trypsin. Following centrifugation, the cells were resuspended in PBS and adjusted to $1 \times 10^{6}$ cells $/ \mathrm{ml}$. According to the instructions of the Annexin V-FITC Apoptosis Detection kit (BD Biosciences), $500 \mu \mathrm{l}$ cell suspension was added into the centrifuge tube at $4^{\circ} \mathrm{C}$ and $150 \mathrm{x}$ for $5 \mathrm{~min}$, followed by the addition of $195 \mu \mathrm{l}$ Annexin V Binding Buffer for resuspension. Then, $5 \mu \mathrm{l}$ Annexin V-FITC and $10 \mu \mathrm{l}$ PI were added into each tube, mixed well and incubated for $10 \mathrm{~min}$ at room temperature in the dark. Cells were evaluated using a MoFlo Astrios EQ flow cytometer (Beckman Coulter, Inc.) admd t Flowjo 7.6 software (FlowJo LLC) was used for data analysis. Total apoptotic rates were calculated as the total early- and late-stage apoptosis.

Light chain 3 (LC3) fluorescence tracer. LC3 double-labeled autophagy adenovirus [Monomeric red fluorescent protein (mRFP)-green fluorescent protein (GFP)-LC3] was purchased from Hanbio Biotechnology Co., Ltd. Transfected cells were cultured in $24-w e l l$ plates $\left(1 \times 10^{5}\right.$ cells $\left./ \mathrm{ml}\right)$ and incubated with mRFP-GFP-LC3 adenovirus (multiplicity of infection, 100) for $2 \mathrm{~h}$ at $37^{\circ} \mathrm{C}$, when cell confluence reached $70-80 \%$. The cells were washed with PBS and cultivated in DMEM containing $10 \%$ FBS overnight. The transfection efficiency of mRFP-GFP-LC3 adenovirus was evaluated using a BX51 fluorescence microscope (Olympus Corporation). ImageJ Pro Plus 6.0 software (NIH) was used for image analysis and counting the number of GFP and RFP fluorescent punctae. In the merge image, autophagosomes are represented by yellow dots and autolysosomes are represented by red dots.

RNA pull-down assay. A431 cells in logarithmic growth phase were collected and centrifuged at $4^{\circ} \mathrm{C}$ and $150 \mathrm{x}$ g for $5 \mathrm{~min}$ and then mixed with Pierce IP Lysis Buffer (Thermo Scientific, Inc.). After lysis on ice for $30 \mathrm{~min}$, the cells were centrifuged at $4^{\circ} \mathrm{C}$ and $1,200 \mathrm{x}$ f for $30 \mathrm{~min}$ to collect the supernatant and stored on ice. RNA-RNA interaction was assessed by RNA pull-down using Pierce Magnetic RNA-Protein Pull-Down kit (Thermo Fisher Scientific, Inc.) following the manufacturer's protocol. Biotinylated HCP5 and NC probes $(50 \mathrm{pmol}$; made using the Pierce RNA 3' End Desthiobiotinylation Kit; cat. no. 20163; Thermo Fisher Scientific, Inc.) were dissolved in washing/binding buffer (contained in the pull-down kit) and cultured with streptavidin-coupled magnetic beads for $2 \mathrm{~h}$ at $4^{\circ} \mathrm{C}$. Next, the probes were added to the cell lysates for $2 \mathrm{~h}$ to elute the RNA complex conjugated with the magnetic beads, and miR expression was determined using RT-qPCR.

Dual luciferase reporter gene assay. The binding sites of 1ncRNA-HCP5 and miR-138-5p, and for EZH2 and miR-138-5p were predicted using RNA22 v2 (https://cm.jefferson.edu/rna22/Interactive) and TargetScan (http://www.targetscan.org) online databases. The HCP5 fragment containing the binding site of miR-138-5p was cloned into the pmirGLO oligosaccharide enzyme vector (Promega
Corporation) to construct the pmirGLO-HCP5-wild-type (WT), and the pmirGLO-HCP5-mutant type (MUT) vector was constructed using the mutant binding site of miR-138-5p. The pmirGLO-EZH2-WT and pmirGLO-EZH2-MUT vectors were constructed using the same methods. The sequences were synthesized by Shanghai GenePharma Co., Ltd. The constructed vectors (50 ng) were transfected into A431 cells (at $80-90 \%$ confluence), and then co-transfected with miR-138-5p or miR-NC (20 nM) according to the instructions of Lipofectamine ${ }^{\circledR} 3000$ (Invitrogen; Thermo Fisher Scientific, Inc.). After incubation at $37^{\circ} \mathrm{C}$ for $48 \mathrm{~h}$, the luciferase activity was evaluated using a Dual Luciferase Reporter Assay System (Promega Corporation), and the relative activity was determined as the ratio of firefly luciferase activity to Renilla luciferase activity.

Animal experiments. A total of 20 specific pathogen-free

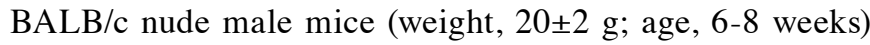
were provided by Beijing Vital River Laboratory Animal Technology Co., Ltd. [license SYXK (Beijing) 2015-0001], numbered according to body weight and allocated into three groups ( $\mathrm{n}=10 \mathrm{mice} / \mathrm{group}$ ) using the random number method. All mice were reared in an animal room at $25^{\circ} \mathrm{C}$ temperature and $50-60 \%$ humidity under a $12 \mathrm{~h} \mathrm{light/dark} \mathrm{cycle.} \mathrm{All} \mathrm{mice}$ were allowed free access to food and water. In the A431 group, each mouse was injected subcutaneously (on the back near the right armpit) with $2 \times 10^{6} \mathrm{~A} 431$ cells. In the si-HCP5-1 group, each mouse was injected subcutaneously with $2 \times 10^{6}$ A431 cells transfected with si-HCP5-1. Tumor volumes were measured every 3 days, and mice were euthanized using pentobarbital (>200 mg/kg intraperitoneal injection) 28 days later $(19,20)$. Death was confirmed by examining pupillary dilation and complete cardiac arrest, and the tumors of five random mice from each group were embedded in paraffin and the tumors from the remaining five mice were ground into a homogenate for subsequent experiments.

Immunohistochemical and TUNEL staining. A Histostain-Plus Kit (cat. no. 85-6643; Invitrogen; Thermo Fisher Scientific, Inc.) was used for immunohistochemical staining. Tumor tissue was fixed with $4 \%$ formaldehyde for $6 \mathrm{~h}$ at room temperature and embedded in paraffin. The paraffin-embedded tumor tissue was cut into $3 \mu \mathrm{m}$ sections; the sections were baked at $45^{\circ} \mathrm{C}$ for $3 \mathrm{~h}$. After dewaxing with xylene and rehydration, the sections were treated with $3 \% \mathrm{H}_{2} \mathrm{O}_{2}$ and kept at room temperature for $10 \mathrm{~min}$ to eliminate the activity of endogenous peroxidase. After washing with PBS $+0.05 \%$ Tween-20 (PBST) three times, the sections were placed in a heat-resistant glass container, submerged in the citrate sodium buffer (10 mM, pH 6.0), heated to boiling in the microwave for antigen retrieval and cooled naturally (5-10 min). These steps were repeated three times and then the sections were cooled down to room temperature. The sections were blocked with $10 \%$ goat serum (reagent $\mathrm{A}$ in the kit) at $37^{\circ} \mathrm{C}$ for $1 \mathrm{~h}$ in a humidified chamber and incubated with anti-Ki67 primary antibody (1:500; cat. no. ab15580; Abcam) at $4^{\circ} \mathrm{C}$ overnight. The next day, sections were washed three times with PBST and incubated with biotinylated secondary antibody working solution (reagent $\mathrm{B}$ in the kit), incubated at $37^{\circ} \mathrm{C}$ for $15 \mathrm{~min}$ and washed three times with PBST times to wash away the 

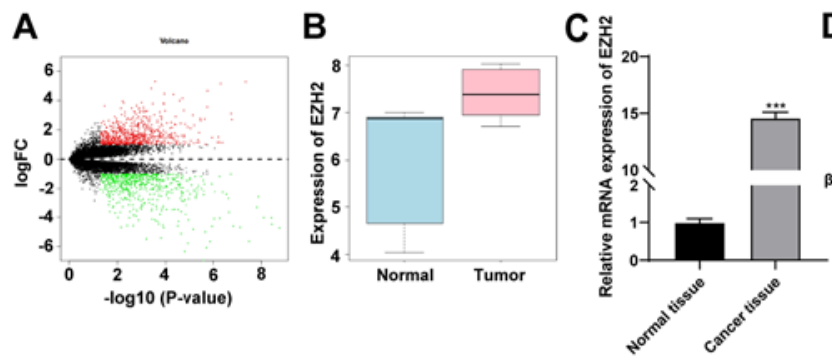

$\mathbf{F}$

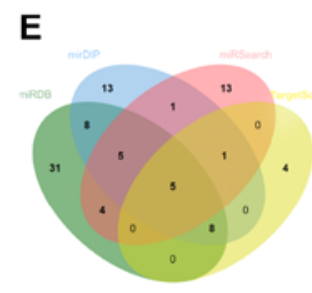

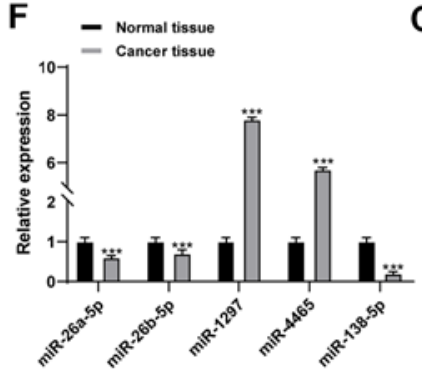

D

G

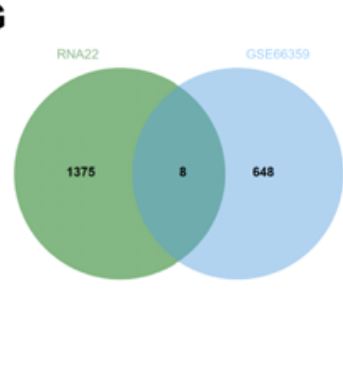

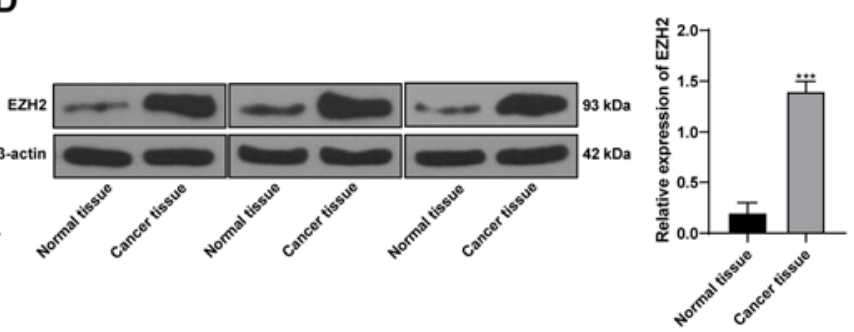

H

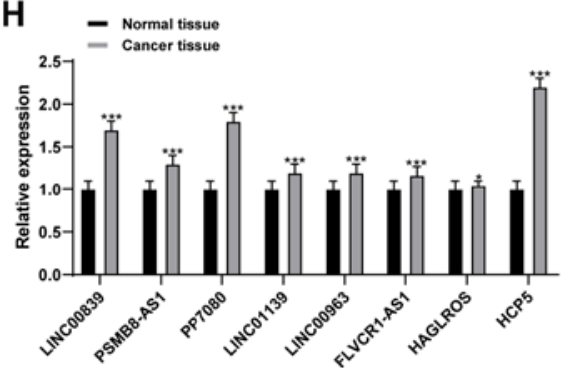

Figure 1. LncRNA HCP5 may regulate EZH2 expression through miR-138-5p. (A) Differential expression analysis of the volcano plot of the GSE66359 microarray. Red indicates highly expressed genes and green indicates genes with low expression levels. (B) EZH2 was found to be significantly increased in CSCC tissue compared with normal tissue in the GSE66359 data set. (C) mRNA and (D) protein expression levels of EZH2 in tissue and normal adjacent tissue samples from 60 patients with CSCC were upregulated. (E) Four online databases were used to analyze the intersected data to predict putative miRNAs upstream of EZH2; five strong candidates were identified: miR-26a-5p, miR-26b-5p, miR-1297, miR-4465 and miR-138-5p. (F) RT-qPCR was used to detect the expression levels of the five miRNAs; miR-138-5p level was the lowest in CSCC compared with normal tissues. $\mathrm{N}=60$; ${ }^{* * *} \mathrm{P}<0.001$ vs. normal tissue. (G) Eight lncRNAs were predicted to regulate miR-138-5p using RNA22 database, and these were intersected with the upregulated genes expressed in GSE66359 microarray $(\mathrm{H})$ The expression of these eight lncRNAs was detected using RT-qPCR, and HCP5 showed the largest upregulation in CSCC compared with normal tissues. $\mathrm{N}=60 ;{ }^{*} \mathrm{P}<0.05,{ }^{* * *} \mathrm{P}<0.001$ vs. normal tissue. Data in panel $\mathrm{B}$ were analyzed using the Wilcoxon signed-rank test, and data in panels $\mathrm{C}$, $\mathrm{D}, \mathrm{F}$ and $\mathrm{H}$ were analyzed using paired Student's t-test. CSCC, cutaneous squamous cell carcinoma; EZH2, enhancer of zeste homolog 2; FC, fold change; HCP5, human histocompatibility leukocyte antigen complex P5; lncRNA, long non-coding RNA; miR, microRNA; RT-qPCR, reverse transcription-quantitative PCR.

unbound antibody. The sections were subsequently incubated with horseradish peroxidase-conjugated streptavidin working solution (reagent $\mathrm{C}$ in the kit), incubated in a wet box at $37^{\circ} \mathrm{C}$ for $15 \mathrm{~min}$, and washed with PBST three times. The percentage of Ki67-positive cells was counted using a CellInsight laser confocal microscope (Thermo Fisher Scientific, Inc.) and analyzed using ImageJ-Pro Plus 6.0 (NIH).

Tumor sections were treated and stained with TUNEL reaction mixture in accordance with the manufacturer's instructions (Wanleibio Co., Ltd.). DAPI solution was added to the media (1:10) to stain the nuclei, and the cells were cultured at $37^{\circ} \mathrm{C}$ for $15 \mathrm{~min}$. Sections were washed with PBS, observed under a fluorescence microscope, and an anti-fluorescence quenching agent (Wanleibio Co., Ltd.) was added. Within $24 \mathrm{~h}$, images of the sections were captured under a fluorescence microscope; the apoptotic cells were counted using ImageJ Pro Plus 6.0 (NIH), and the results were analyzed.

Statistical analysis. Statistical analyses were conducted using SPSS v21.0 (IBM Corp.). The Kolmogorov-Smirnov test confirmed that the data were normally distributed. Data are presented as the mean \pm standard deviation. Paired Student's t-test or Mann-Whitney U test was used for comparisons between two groups, whereas one-way ANOVA was used to compare multiple groups; Tukey's multiple comparisons test was applied for pairwise comparisons after ANOVA analyses. The P-value was obtained using a two-tailed test, and $\mathrm{a}<0.05$ was used to indicate a statistically significance difference.
Table IV. Potential lncRNAs regulating miR-138-5p.

\begin{tabular}{lcc}
\hline lncRNA & llogFCl & P-value \\
\hline LINC00839 & 1.535958914 & $3.05 \times 10^{-5}$ \\
PSMB8-AS1 & 1.418830957 & $5.94 \times 10^{-5}$ \\
PP7080 & 1.660170378 & $5.96 \times 10^{-4}$ \\
LINC01139 & 1.218616424 & $1.065 \times 10^{-3}$ \\
LINC00963 & 1.129432649 & $1.333 \times 10^{-3}$ \\
FLVCR1-AS1 & 1.170397507 & $3.735 \times 10^{-3}$ \\
HAGLROS & 1.030294427 & $4.908 \times 10^{-3}$ \\
HCP5 & 1.800169542 & $6.281 \times 10^{-3}$ \\
\end{tabular}

AS, antisense RNA; lncRNA, long non-coding RNA; miR, microRNA; HCP5, histocompatibility leukocyte antigen complex P5.

\section{Results}

HCP5 and EZH2 are highly expressed and miR-138-5p expression is low in CSCC. Through the differential analysis of GSE66359 microarray, 1,252 genes with significant differential expression were obtained (Fig. 1A). Among these genes, EZH2 was screened based on a literature search that suggested EZH2 can be used as a diagnostic index to differentiate squamous cell carcinoma from normal skin (14). EZH2 expression was significantly increased in CSCC compared with normal tissue (Fig. 1B), which was consistent with a previous report (14). EZH2 mRNA and protein 

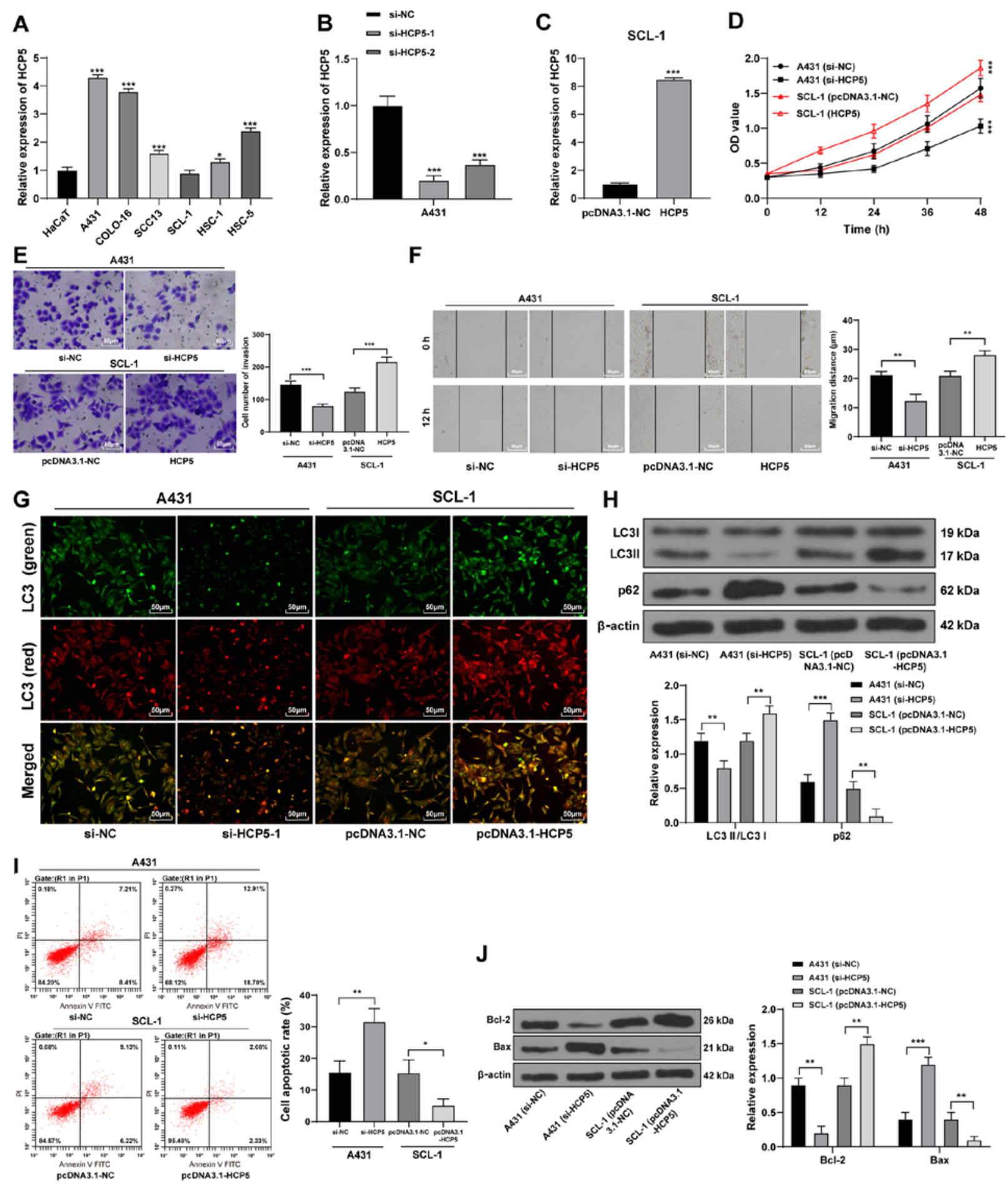

Figure 2. Long non-coding RNA HCP5 regulates the malignant behavior, autophagy and apoptosis of CSCC cells. (A) RT-qPCR detection of HCP5 expression in CSCC cell lines. ${ }^{*} \mathrm{P}<0.05,{ }^{* * *} \mathrm{P}<0.001$ vs. HaCaT. The transfection efficiency of (B) two siRNAs against HCP5 and (C) the HCP5 overexpression vector were verified using RT-qPCR. ${ }^{* * *} \mathrm{P}<0.001$ vs. si-NC or pcDNA3.1-NC. (D) Cell Counting Kit-8 assays, (E) Transwell assays and (F) scratch tests were used to detect the cell viability, migration and invasion, respectively. ${ }^{* *} \mathrm{P}<0.01,{ }^{* * *} \mathrm{P}<0.001$ vs. si-NC or pcDNA3.1-NC. (G) Representative images of mRFP-GFP-LC3 fluorescence tracer assay, in which green and red indicate the observed fluorescence of LC3 under different excitation wavelengths; yellow fluorescence indicates autophagosomes and autolysosomes are represented by red dots. $(\mathrm{H})$ Western blot analysis was used to detect the levels of autophagy-related proteins. ${ }^{* *} \mathrm{P}<0.01$, ${ }^{* * * *} \mathrm{P}<0.001$ vs. si-NC or pcDNA3.1-NC. (I) Flow cytometry was used to determine the apoptotic rates in transfected CSCC cells. ${ }^{*} \mathrm{P}<0.05$, ${ }^{* *} \mathrm{P}<0.01$ si-NC or pcDNA3.1-NC. (J) Western blot analysis was used to detect the levels of apoptosis-related proteins. ${ }^{* *} \mathrm{P}<0.01,{ }^{* * *} \mathrm{P}<0.001$ vs. si-NC or pcDNA3.1-NC. Data in panels A, B and D were analyzed using one-way ANOVA, followed by Tukey's multiple comparisons test, and data in panels C, E, F, H, I and J were analyzed using the Mann-Whitney U-test. Data are representative of three experimental repeats. GFP green fluorescent protein; HCP5, human histocompatibility leukocyte antigen complex P5; LC3, light chain 3; mRFP, monomeric red fluorescent protein; NC, negative control; RT-qPCR, reverse transcription-quantitative PCR; siRNA, small interfering RNA.

expression levels in 60 patients with CSCC collected from The First Affiliated Hospital of Zhengzhou University also verified these results (Fig. 1C and D, respectively). To further understand the mechanism of EZH2 in CSCC, the upstream 

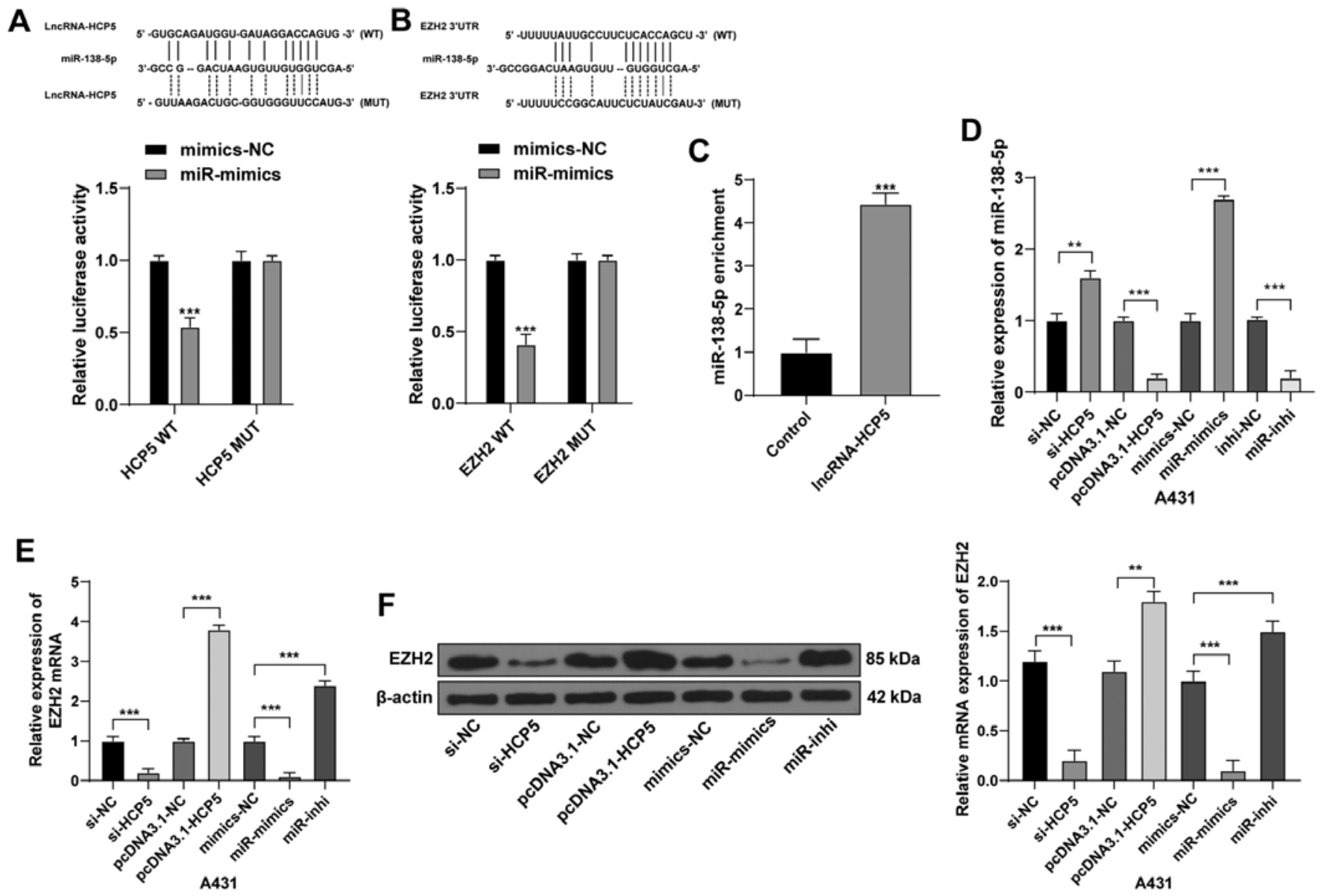

Figure 3. LncRNA HCP5 competes with EZH2 to bind to miR-138-5p. Binding sites between (A) HCP5 and miR-138-5p, and (B) the miR-138-5p target sites in EZH2 3'UTR and were predicted using the online databases RNA22 and TargetScan. Dual luciferase report gene assay verified the relation between (A) HCP5 and miR-138-5p and (B) EZH2 and miR-138-5p. ${ }^{* * * *} \mathrm{P}<0.001$ vs. mimics-NC. (C) miR-138-5p was significantly enriched by HCP5 using RNA pull-down assay. ${ }^{* * * *} \mathrm{P}<0.001$ vs. Control. (D) miR-138-3p, (E) EZH2 mRNA and (F) EZH2 protein expression levels in A431 cells following the various transfections were detected using reverse transcription-quantitative PCR or western blotting. ${ }^{* *} \mathrm{P}<0.01,{ }^{* * * *} \mathrm{P}<0.001$. Data are representative of three experimental repeats. Data were analyzed by Mann-Whitney U-test. EZH2, enhancer of zeste homolog 2; HCP5, human histocompatibility leukocyte antigen complex P5; inhi, inhibitor; lncRNA, long non-coding RNA; miR, microRNA; MUT, mutant; UTR, untranslated region; WT, wild-type.

regulatory miRNAs of $E Z H 2$ were predicted using four online databases. The combined prediction results identified five miRNAs that may target EZH2 (Fig. 1E). RT-qPCR was used to determine the expression levels of the five miRNAs, which revealed that the relative miR-138-5p expression was the lowest in CSCC compared with its expression in normal tissue (Fig. 1F). miR-138-5p has been shown to inhibit tumor development $(21,22)$, but its role in CSCC remains unclear. Using the RNA22 database, lncRNAs that may interact with miR-138-5p were predicted. The prediction results of RNA22 were intersected with the upregulated gene expression results obtained from GSE66359 microarray (Fig. 1G), and eight putative lncRNAs were identified that may regulate miR-138-5p; these IncRNAs were shown to be highly expressed in CSCC compared with normal adjacent tissue (Fig. 1H). Furthermore, the expression of these eight lncRNAs were screened in the GSE66359 microarray (Table IV) and found that HCP5 had the greatest upregulation in CSCC $(\mid \log F C l=1.8)$ and the highest relative expression levels in patient CSCC compared with the normal adjacent tissue (Fig. 1H). These data suggested that IncRNA HCP5 may regulate EZH2 expression through miR-138-5p.
Silencing HCP5 inhibits malignant behaviors and autophagy of CSCC cells and promotes apoptosis. The expression levels of IncRNA HCP5 were detected in cultured CSCC cell lines and the HaCaT human immortalized keratinocyte cell line. A431 cells exhibited the highest relative HCP5 expression and SCL-1 cells exhibited the lowest HCP5 expression levels among the CSCC cell lines (Fig. 2A); therefore, these two lines were used for subsequent experiments. Two siRNAs against HCP5-1 were transfected into A431 cells, of which si-HCP5-1 exhibited the best transfection efficiency and was selected for the following experiment (Fig. 2B). An HCP5 overexpression vector was transfected into SCL-1 cells, and the transfection efficiency was verified using RT-qPCR (Fig. 2C). Silencing of HCP5 expression resulted in significant decreases in A431 cell viability, invasion and migration, whereas SCL-1 cells overexpressing HCP5 exhibited opposite trends (Fig. 2D-F).

LncRNAs can regulate apoptosis and autophagy of CSCC cells (23). Therefore, autophagy and apoptosis of CSCC cells were measured following HCP5 knockdown and overexpression. After HCP5 silencing, the number of autophagosomes (yellow punctae) decreased (Fig. 2G), the LC3II/LC3I protein expression ratio decreased and the 
A

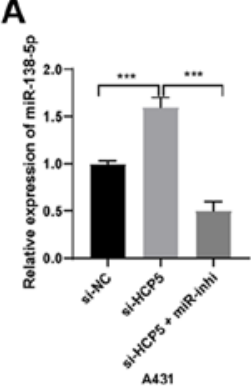

D

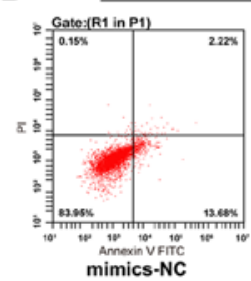

E

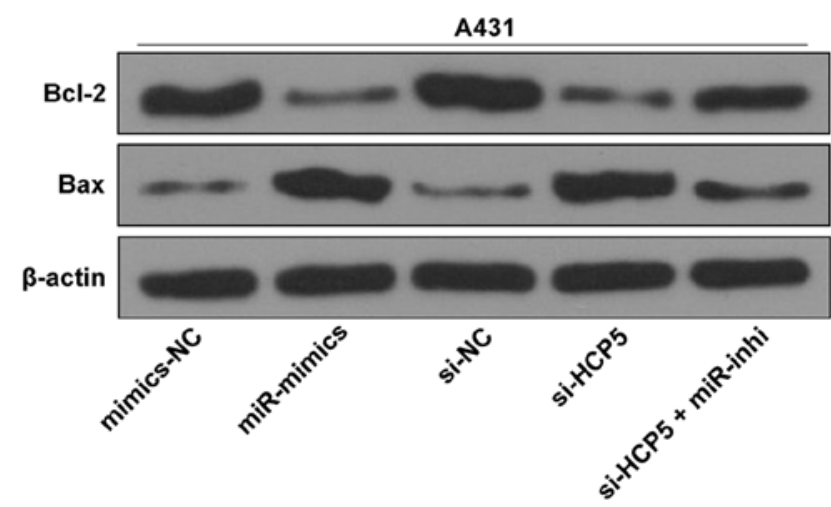

B

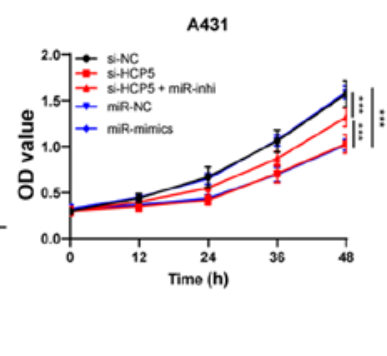

C

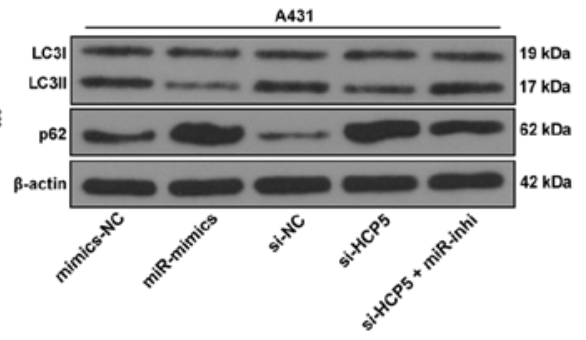

A431

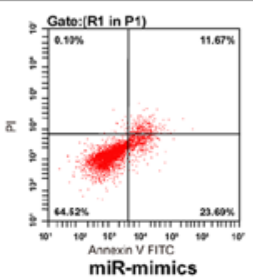

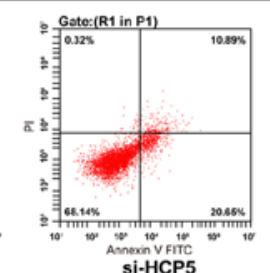
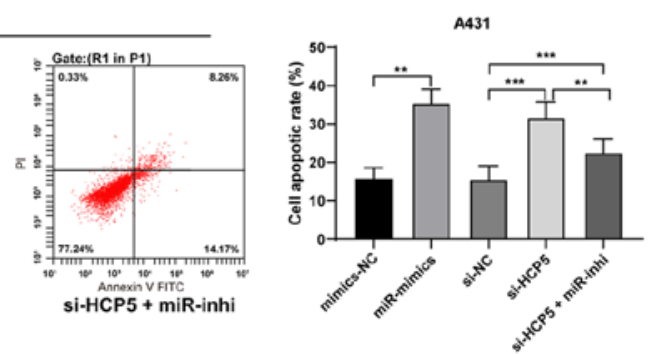

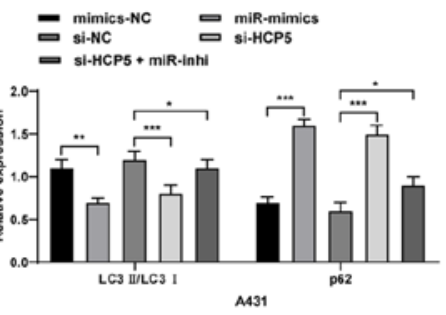

A43

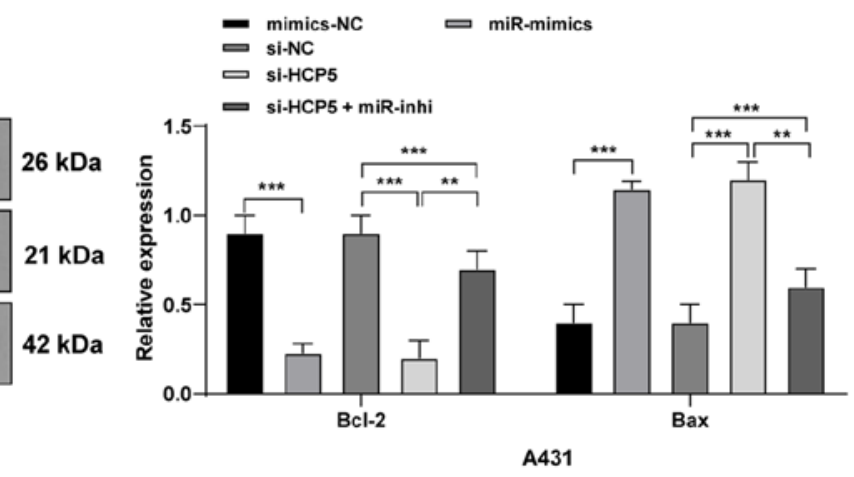

Figure 4. Upregulation of miR-138-5p in cutaneous squamous cell carcinoma cells inhibits autophagy and promotes apoptosis. (A) miR-138-5p inhibitor was co-transfected into A431 cells with si-HCP5, and the expression levels of miR-138-5p were detected by reverse transcription-quantitative PCR. ${ }^{* * *} \mathrm{P}<0.001$. (B) Cell Counting Kit-8 assay was used to measure cell viability at each time point. ${ }^{* * *} \mathrm{P}<0.001$. (C) Western blot analysis was used to determine the expression levels of autophagy-related proteins. ${ }^{*} \mathrm{P}<0.05,{ }^{* *} \mathrm{P}<0.01,{ }^{* * *} \mathrm{P}<0.001$. (D) Flow cytometry was used to determine the apoptotic rates in transfected cells. ${ }^{* *} \mathrm{P}<0.01$, ${ }^{* * *} \mathrm{P}<0.001$. (E) Western blot analysis was used to determine the expression levels of apoptosis-related proteins. ${ }^{* *} \mathrm{P}<0.01,{ }^{* * *} \mathrm{P}<0.001$. Data are representative of three experimental repeats. Data in panels A and E were analyzed by Mann-Whitney U-test, and data in panels B-D were analyzed using the one-way ANOVA followed by Tukey's multiple comparisons test. EZH2, enhancer of zeste homolog 2; HCP5, human histocompatibility leukocyte antigen complex P5; inhi, inhibitor; LC3, light chain 3; miR, microRNA; NC, negative control; si, small interfering RNA.

expression of p62 protein increased (Fig. 2H). In addition, the apoptotic rate significantly increased (Fig. 2I), apoptotic protein Bax expression level increased and Bcl-2 expression level significantly decreased (Fig. 2J). Conversely, the number of autophagosomes in CSCC cells increased following HCP5 overexpression, the protein expression levels of LC3 II/LC3I increased, whereas the expression of p62, the apoptotic rate and Bax protein expression decreased; Bcl-2 expression increased significantly. In summary, HCP5 silencing inhibited the malignant behaviors and autophagy of CSCC cells and promoted apoptosis, whereas overexpression of HCP5 promoted the malignant behaviors and autophagy of CSCC cells and inhibited apoptosis.

HCP5 competes with EZH2 to bind to miR-138-5p. Combined with the microarray analysis results, the binding sites of 1ncRNA HCP5 and miR-138-5p as well as EZH2 and miR-138-5p were predicted using online databases RNA22 and TargetScan. Dual luciferase reporter assays were used to verify the binding relationships (Fig. 3A-B). In addition, lncRNA HCP5 significantly enriched miR-138-5p using an RNA pull-down assay (Fig. 3C). As shown in Fig. 3D-F, after HCP5 silencing in A431 cells, miR-138-5p expression levels increased, whereas the EZH2 mRNA and protein expression levels decreased significantly; the opposite trends were observed in cells overexpressing HCP5. After miR-138-5p mimics transfection, EZH2 expression levels decreased, whereas the results of miR-138-5p inhibitor transfection were the opposite. These results indicated negative regulatory relationships between HCP5 and miR-138-5p, and between EZH2 and miR-138-5p. HCP5 may function as a ceRNA to compete with EZH2 to bind to miR-138-5p.

Upregulation of miR-138-5p in CSCC cells inhibits autophagy and promotes apoptosis. miR-138-5p inhibitor was co-transfected into A431 cells with si-HCP5 to conduct a functional 


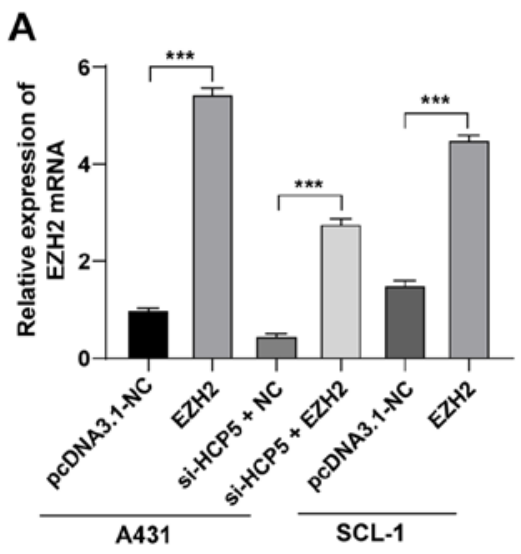

C
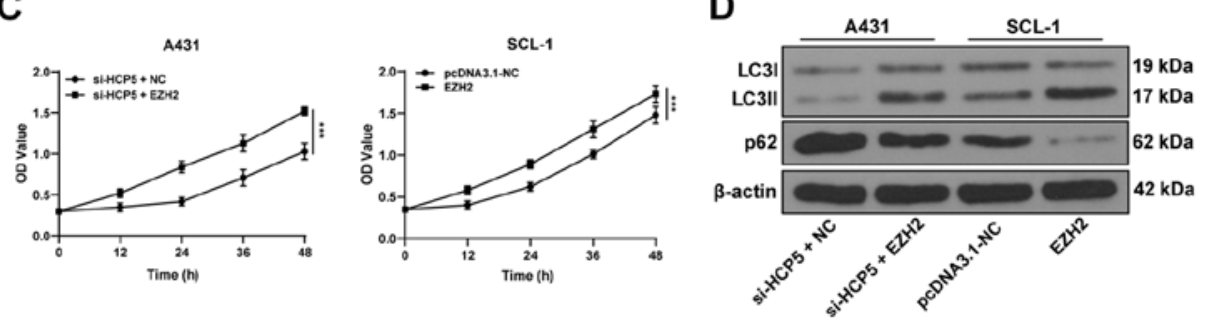
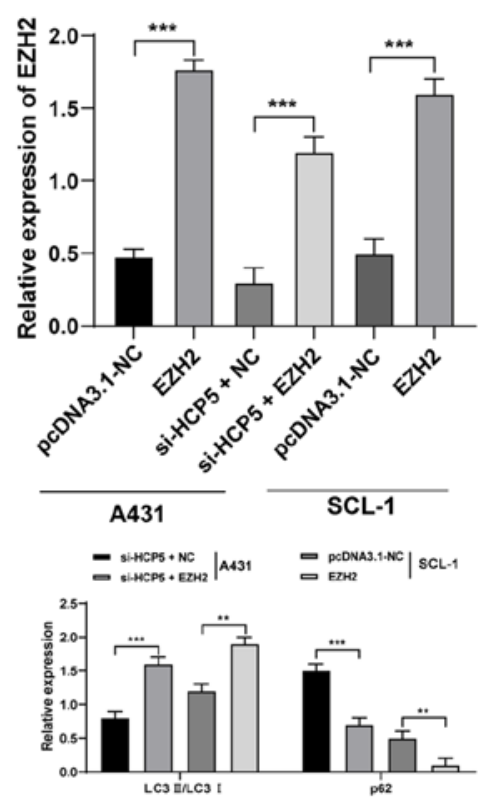

E

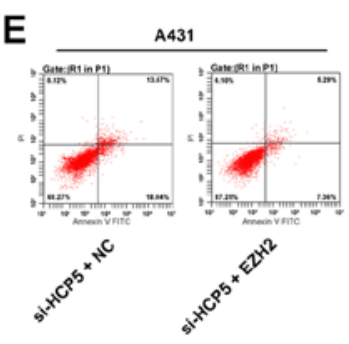

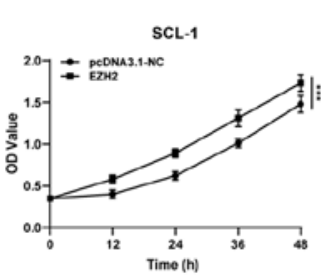

D

B

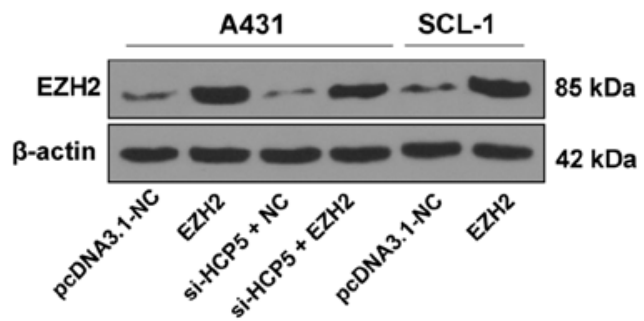

F

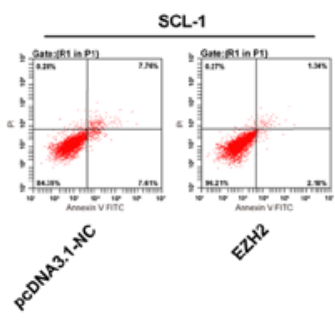

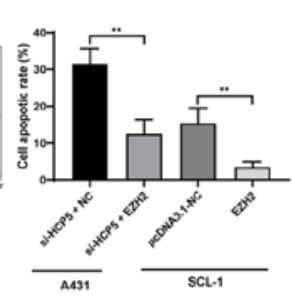

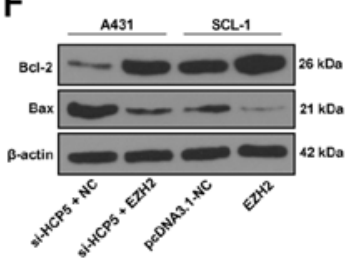

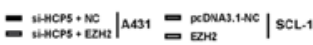

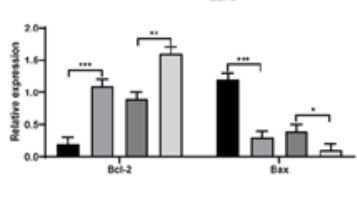

Figure 5. Upregulation of EZH2 counteracts the effects of HCP5 silencing in cutaneous squamous cell carcinoma cells. (A) mRNA and (B) protein expression levels of EZH2 were detected using reverse transcription-quantitative PCR and western blot analysis, respectively. ${ }^{* * *} \mathrm{P}<0.001$. (C) Cell Counting Kit- 8 assays were used to measure cell viability at each time point. ${ }^{* * *} \mathrm{P}<0.001$. (D) Western blot analysis was used to determine the expression levels of autophagy-related proteins. ${ }^{* *} \mathrm{P}<0.01$, ${ }^{* * * *} \mathrm{P}<0.001$. (E) Flow cytometry was used to determine the apoptotic rates in transfected cells. ${ }^{* *} \mathrm{P}<0.01$. (F) Western blot analysis was used to determine the expression levels of apoptosis-related proteins. ${ }^{*} \mathrm{P}<0.05,{ }^{* *} \mathrm{P}<0.01,{ }^{* * *} \mathrm{P}<0.001$. Data in panel $\mathrm{C}$ were analyzed using one-way ANOVA, followed by Tukey's multiple comparisons test, and data in panels A, B and D-F were analyzed using the Mann-Whitney U-test. EZH2, enhancer of zeste homolog 2; HCP5, human histocompatibility leukocyte antigen complex P5; inhi, inhibitor; LC3, light chain 3; miR, microRNA; NC, negative control; si, small interfering RNA.

rescue experiment (Fig. 4A). Cell viability was reduced upon upregulation of miR-138-5p or downregulation of $H C P 5$, whereas downregulation of miR-138-5p partially counteracted the effect of HCP5 silencing on CSCC viability (Fig. 4B). HCP5 silencing and upregulation of miR-138-5p significantly decreased the LC3II/LC3I ratio and increased the protein expression of p62 in CSCC cells; downregulation of miR-138-5p partially reversed the inhibitory effects of HCP5 silencing in CSCC cells (all $\mathrm{P}<0.05$; Fig. 4C). Additionally, si-HCP5 or miR-138-5p mimics alone significantly increased the apoptotic rate, increased the protein expression levels of Bax and decreased Bcl-2; downregulation of miR-138-5p partially abolished the promoting effect of HCP5 silencing on cell apoptosis (Fig. 4D-E). These results indicated that upregulation of miR-138-5p may inhibit autophagy and promote apoptosis of CSCC cells.

Upregulation of EZH2 counteracts the effects of silencing HCP5 on CSCC cells. An EZH2 overexpression vector was transfected into CSCC cells (Fig. 5A and B). EZH2 overexpression increased the viability of SCL-1 cells and offset the inhibitory effect of silencing HCP5 on A431 cell viability
(Fig. 5C). Western blotting analysis revealed that overexpression of EZH2 significantly increased the protein expression ratio of LC3II/LC3I, decreased the expression of p62 in SCL-1 cells and counteracted the inhibitory effects of HCP5 silencing on the autophagy of A431 cells (Fig. 5D). Additionally, overexpression of EZH2 significantly decreased the level of apoptosis (Fig. 5E), decreased the protein expression levels of Bax and increased the expression of Bcl-2 in SCL-1 cells (Fig. 5F), and counteracted the effects of HCP5 silencing in of A431 cells.

EZH2 affects autophagy and apoptosis of CSCC cells through the STAT3/VEGFR2 pathway. EZH2 was previously reported to serve a regulatory role in head and neck squamous cell carcinoma through the STAT3/VEGFR2 pathway (24). Therefore, whether the STAT3/VEGFR2 pathway is involved in CSCC development was investigated. Western blotting results revealed that p-STAT3/total (t)-STAT3 ratio and VEGFR2 protein expression levels in CSCC cells overexpressing EZH2 were significantly increased compared with the control (Fig. 6A). Co-treatment with AG-490, an inhibitor of the STAT3/VEGFR2 pathway, significantly inhibited the levels 
A

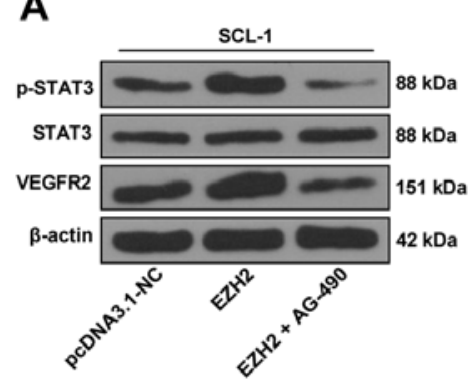

C

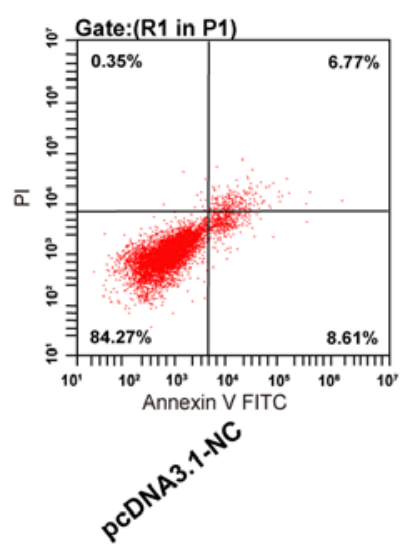

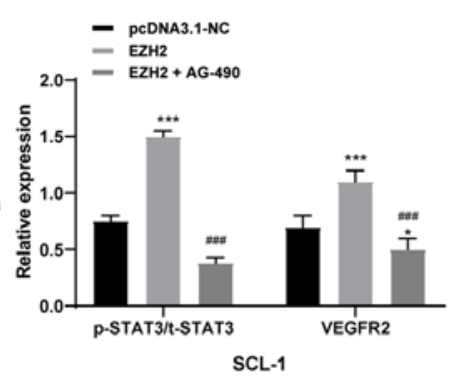

SCL-1
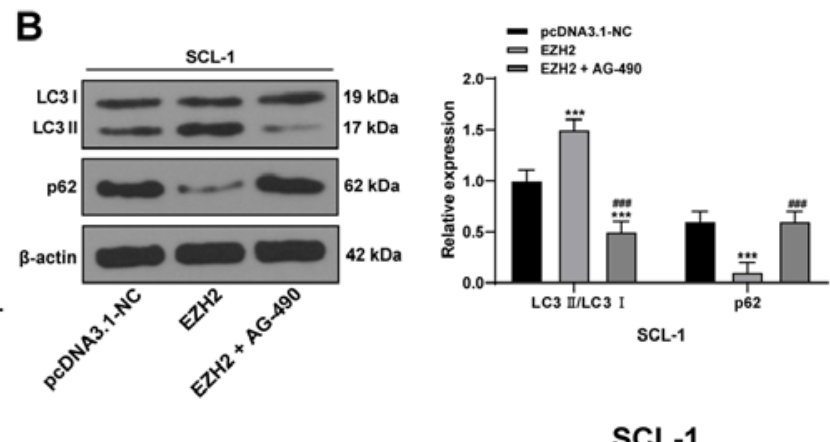

SCL-1
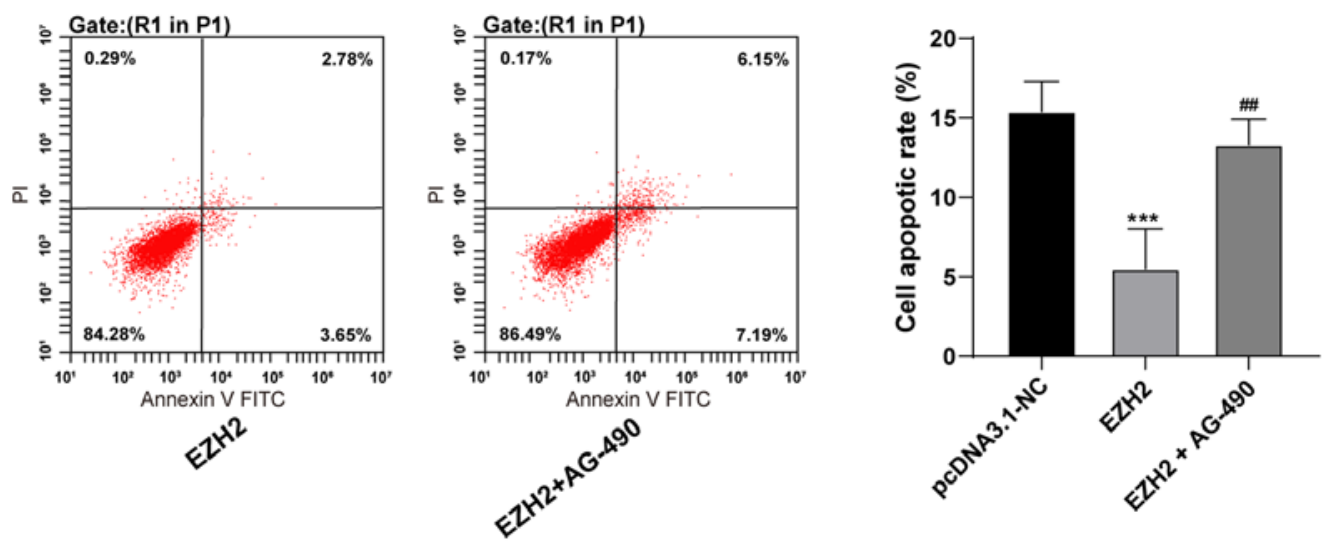

Figure 6. EZH2 regulates autophagy and apoptosis in cutaneous squamous cell carcinoma cells through the STAT3/VEGFR2 pathway. Western blot analysis was used to determine the expression levels of (A) p-STAT3, t-STAT3 and VEGFR2 proteins and (B) autophagy-related proteins. ${ }^{*} \mathrm{P}<0.05$, ${ }^{* * *} \mathrm{P}<0.001$ vs. pcDNA3.1-NC; ${ }^{\# \# ~} \mathrm{P}<0.001$ vs. EZH2. (C) Flow cytometry was used to determine the apoptotic rates in transfected cells. ${ }^{* * *} \mathrm{P}<0.001$ vs. pcDNA3.1-NC; ${ }^{\# \#} \mathrm{P}<0.01$ vs. EZH2. Data are representative of three experimental repeats. Data were analyzed using one-way ANOVA, followed by Tukey's multiple comparisons test. EZH2, enhancer of zeste homolog 2; LC3, light chain 3; NC, negative control; p-, phosphorylated, t-, total.

of p-STAT3/t-STAT3 and VEGFR2 in EZH2-overexpressing CSCC cells (Fig. 6A). In addition, AG-490 co-treatments also resulted in decreased autophagy, as determined by western blot analysis of autophagy-related proteins (Fig. 6B), and the apoptotic rate increased (Fig. 6C). Taken together, EZH2 may regulate autophagy and apoptosis in CSCC cells through the STAT3/VEGFR2 pathway.

HCP5 promotes EZH2 expression to activate the STAT3/VEGFR2 pathway in vivo. si-HCP5 or si-NC A431 cells were subcutaneously injected into mice. Tumor volume and weight measurements indicated that tumor growth was limited after silencing HCP5 compared with the control group (Fig. 7A-C). In addition, the protein expression levels of EZH2, p-STAT3/t-STAT3 ratio and VEGFR2 were decreased; autophagy-related proteins LC3II/LC3I decreased significantly, whereas p62 protein expression increased (Fig. 7D). After HCP5 silencing, the Ki67-positive rate was significantly reduced (Fig. 7E), and TUNEL staining indicated that apoptosis was increased (Fig. 7F). These data suggested that HCP5 may promote EZH2 expression, increase proliferation and autophagy, and reduce apoptosis in CSCC cells through the STAT3/VEGFR2 pathway.

\section{Discussion}

CSCC is an immense clinical and economic problem given its incidence has increased over $200 \%$ in the last three decades $(25,26)$. The mortality of high-risk and metastatic CSCC is $40 \%$ within
3 years (27). Therefore, early diagnosis and treatment of CSCC is crucial to minimize morbidity and save medical resources (2). In the present study, differentially expressed mRNAs, miRNAs and IncRNAs in CSCC samples were identified, a putative EZH2/miR-138-5p/lncRNA HCP5 ceRNA regulatory network was predicted, and their effects on autophagy and apoptosis in CSCC cells were investigated. The data suggested that HCP5 competitively binds miR-138-5p to regulate EZH2 in CSCC cells and promoted autophagy and reduced the level of apoptosis through the STAT3/VEGFR2 pathway.

Using GSE66359 microarray analysis, database prediction and verification in $60 \mathrm{CSCC}$ tissues, it was revealed that $\mathrm{EZH} 2$ and HCP5 were highly expressed in CSCC cells, whereas miR-138-5p expression was low. EZH2 is increased in skin cancer cell lines, and the knockdown of EZH2 is associated with a decreased formation of histone H3 lysine 27 trimethylation and a reduction in survival (28). EZH2 expression may be used as a diagnostic marker for differentiating CSCC from actinic keratosis or normal skin (14). miR-138-5p is markedly downregulated in oral squamous cell carcinoma (29). HCP5 is overexpressed in cervical cancer (30), follicular thyroid carcinoma (10) and colon cancer (31). However, the expression profile and molecular mechanism of HCP5 in CSCC remain unknown. Therefore, the next focus of this study was to explore the role of HCP5 in malignant behaviors in CSCC cells.

The second major result of the present study was that, after silencing HCP5, the LC3 autophagosomes, LC3II/LC3I and Bcl-2 levels decreased, whereas the apoptotic rate and the expression levels of p62 and the apoptosis-related protein Bax 
A

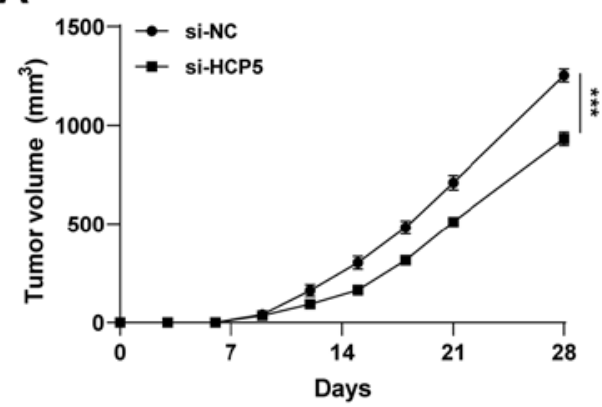

B
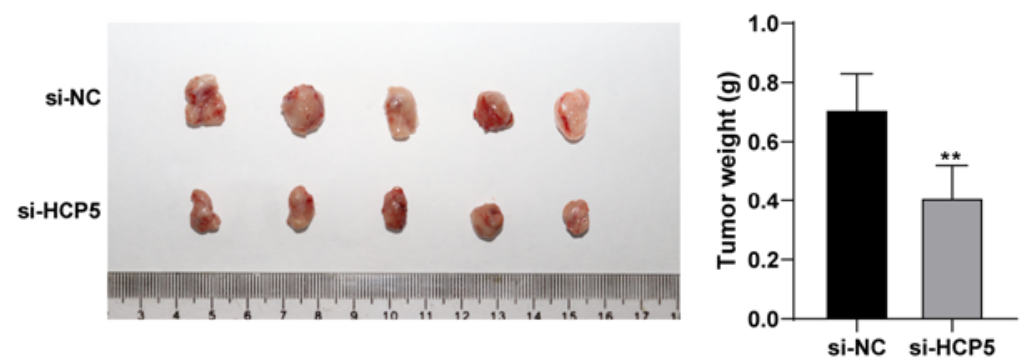

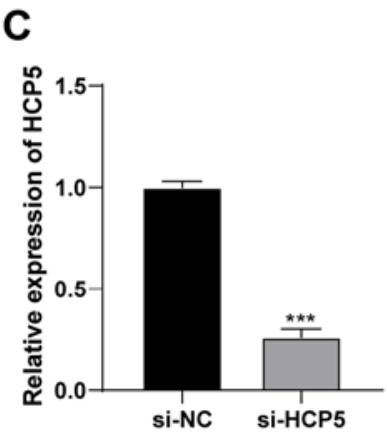

D
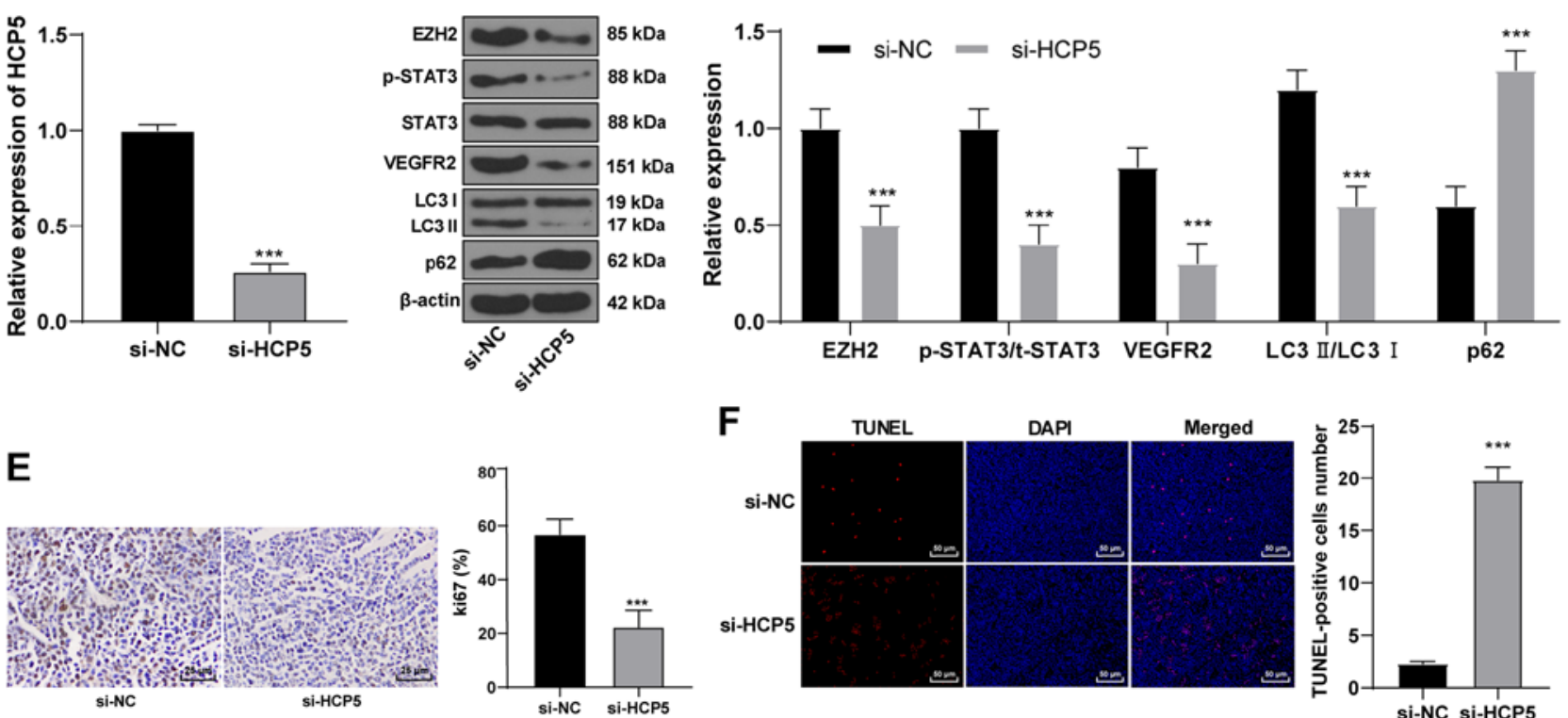

F
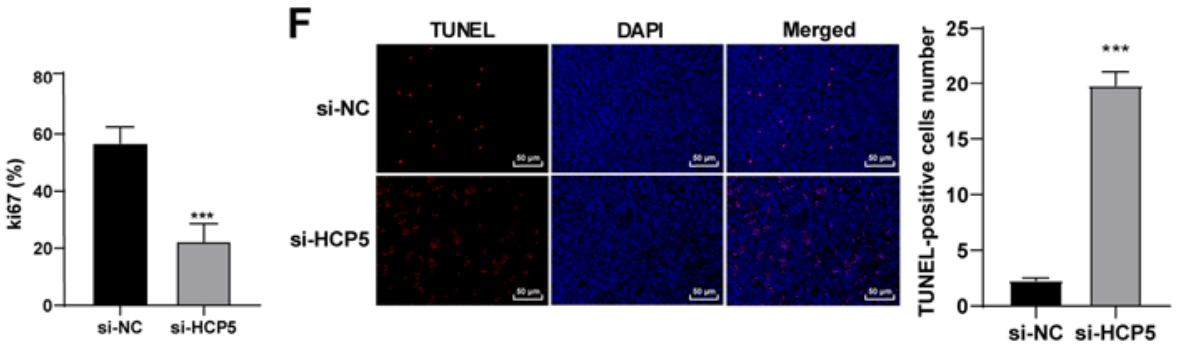

Figure 7. HCP5 promotes EZH2 expression to activate the STAT3/VEGFR2 pathway in vivo. Tumor (A) volume and (B) weight after HCP5 silencing in nude mice. ${ }^{* *} \mathrm{P}<0.01,{ }^{* * * *} \mathrm{P}<0.001$ vs. si-NC. (C) HCP5 expression levels in the excised tumors were detected using reverse transcription-quantitative $\mathrm{PCR}$. ${ }^{* * *} \mathrm{P}<0.001$ vs. si-NC. (D) Western blot analysis was used to determine the expression levels of the p-STAT3, t-STAT3 and VEGFR2 proteins and autophagy-related proteins. ${ }^{* * *} \mathrm{P}<0.001$ vs. si-NC. (E) Immunohistochemistry was used to detect the Ki67-positive expression rate. ${ }^{* * *} \mathrm{P}<0.001$ vs. si-NC. (F) TUNEL staining was used to detect cell apoptosis in tumors. ${ }^{* * *} \mathrm{P}<0.001$ vs. si-NC. N=5. Data in panel A were analyzed using one-way ANOVA, followed by Tukey's multiple comparisons test, and data in panels B-E were analyzed using Mann-Whitney U-test. EZH2, enhancer of zeste homolog 2; HCP5, human histocompatibility leukocyte antigen complex P5; LC3, light chain 3; NC, negative control; p-, phosphorylated; si, small interfering RNA; t-, total.

level increased significantly. Opposite results were obtained for cells overexpressing HCP5. The overexpression of LC3 protein is also strongly correlated with metastasis and a poor clinical prognosis of human melanoma (32). Impaired autophagy leads to p62 accumulation (33). High expression of LC3 and low levels of p62 may play key roles in CSCC tumorigenesis and metastasis (5). In a previous study, Wright et al (34) reported that with increased CSCC stage, the basic level of LC3-II tripled, and that autophagy inhibition enhanced the sensitivity of metastatic CSCC cells to docetaxel-induced apoptosis. HCP5 upregulation was also observed in glioma, and HCP5 knockdown blocked proliferation and invasion, and promoted apoptosis (11). In the present study, silencing of HCP5 inhibited migration and invasion and autophagy in CSCC cells and promoted apoptosis. Additionally, using database prediction, a dual luciferase reporter gene assay system, and an RNA pull-down assay, HCP5 was demonstrated to compete with EZH 2 to bind to miR-138-5p. A recent study uncovered the ceRNA network involving lncRNA HCP5 in pancreatic cancer, in which HCP5 serves as a sponge for miR-214-3p to target hepatoma-derived growth factor to modulate cell apoptosis, autophagy and drug resistance (35).

Furthermore, the present study data revealed that miR-138-5p upregulation in CSCC cells inhibited autophagy and promoted apoptosis. Reduced miR-138 levels are related to poor clinical conditions in patients with esophageal and oral SCC $(36,37)$. Similarly, miR-138-5p substantially reduced the expression levels of autophagy markers in pancreatic cancer cells and impaired serum starvation-driven autophagy flux (38). miR-138-5p inhibited the migratory and invasive capacity by regulating epithelial-mesenchymal transition (EMT) in head and neck SCC cell lines (39). In the present study, EZH2 upregulation counteracted the effects of HCP5 silencing on CSCC cells. EZH2 downregulation was reported to repress inflammation and metastases, and prevented tumor dissemination in CSCC (40). EZH2 knockdown has also shown to reduce the autophagic activity and proliferation of laryngeal SCC cells (15). 
Regarding the HCP5/miR-138-5p/EZH2 ceRNA mechanism in CSCC cells, the present study explored the potential downstream pathways involved in CSCC development. EZH2 serves a regulatory role in the head and neck SCC process through the STAT3/VEGFR2 pathway (24). STAT3 inhibitor treatment suppresses EMT, proliferation and migration of A431 CSCC cells (41). EGFR and STAT3 are frequently overexpressed in CSCC cell lines and tissues (42). VEGFR2 has also been shown to boost tumor proliferation in CSCC cells (43). In other words, EZH2 affects autophagy and apoptosis of CSCC cells through the STAT3/VEGFR2 pathway.

In conclusion, the present study investigated the ceRNA network of lncRNA HCP5/miR-138-5p/EZ2H in CSCC. We hypothesized that the silencing of $H C P 5$ downregulated EZ2H and inactivated the STAT3/VEGFR2 pathway by competitively binding to miR-138-5p, thus inhibiting autophagy and promoting the apoptosis of CSCC cells. A detailed study on the effects of HCP5 on the proliferative ability of CSCC cells will be conducted in the follow-up research. The present results may provide a new perspective to evaluate the function of the HCP5/miR-138-5p/EZ2H axis in CSCC, and thus may offer cancer prevention and therapeutic strategies. Further investigations should be performed to validate these data and to determine their clinical value.

\section{Acknowledgements}

Not applicable.

\section{Funding}

No funding was received.

\section{Availability of data and materials}

All data generated or analyzed during this study are included in this published article.

\section{Authors' contributions}

SBZ contributed to the study design and manuscript preparation. YG contributed to the experimental studies and data acquisition. STZ contributed to the data analysis and statistical analysis. All authors read and approved the final manuscript.

\section{Ethics approval and consent to participate}

This study was performed with the approval of The Clinical Ethical Committee of The First Affiliated Hospital of Zhengzhou University [Zhengzhou, China; (KY-2021-0036)]. All patients agreed to participate and signed the informed consent. Ethics approval was also received from the animal ethics committee of The First Affiliated Hospital of Zhengzhou University (2021-KY-0107-001). All procedures were conducted in accordance with the code of ethics. Great efforts were made to minimize the number of animals used and their pain.

\section{Patient consent for publication}

Not applicable.

\section{Competing interests}

The authors declare that they have no competing interests.

\section{References}

1. Que SKT, Zwald FO and Schmults CD: Cutaneous squamous cell carcinoma: Incidence, risk factors, diagnosis, and staging. J Am Acad Dermatol 78: 237-247, 2018.

2. Parekh V and Seykora JT: Cutaneous squamous cell carcinoma. Clin Lab Med 37: 503-525, 2017.

3. Brinkman JN, Hajder E, van der Holt B, Den Bakker MA, Hovius SE and Mureau MA: The effect of differentiation grade of cutaneous squamous cell carcinoma on excision margins, local recurrence, metastasis, and patient survival: A retrospective follow-up study. Ann Plast Surg 75: 323-326, 2015.

4. Thompson AK, Kelley BF, Prokop LJ, Murad MH and Baum CL: Risk factors for cutaneous squamous cell carcinoma recurrence, metastasis, and disease-specific death: A systematic review and meta-analysis. JAMA Dermatol 152: 419-428, 2016.

5. Zheng LQ, Li SY and Li CX: Expression profiling analysis of autophagy-related genes in perineural invasion of cutaneous squamous cell carcinoma. Oncol Lett 15: 4837-4848, 2018.

6. Ou C, Liu H, Ding Z and Zhou L: Chloroquine promotes gefitinib-induced apoptosis by inhibiting protective autophagy in cutaneous squamous cell carcinoma. Mol Med Rep 20: 4855-4866, 2019.

7. Lu MY, Liao YW, Chen PY, Hsieh PL, Fang CY, Wu CY, Yen ML, Peng BY, Wang DP, Cheng HC, et al: Targeting LncRNA HOTAIR suppresses cancer stemness and metastasis in oral carcinomas stem cells through modulation of EMT. Oncotarget 8: 98542-98552, 2017.

8. Sand M, Bechara FG, Sand D, Gambichler T, Hahn SA, Bromba M, Stockfleth E and Hessam S: Expression profiles of long noncoding RNAs in cutaneous squamous cell carcinoma. Epigenomics 8: 501-518, 2016.

9. Kulski JK: Long Noncoding RNA HCP5, a Hybrid HLA Class I endogenous retroviral gene: Structure, expression, and disease associations. Cells 8: 480, 2019.

10. Liang L, Xu J, Wang M, Xu G, Zhang N, Wang G and Zhao Y: LncRNA HCP5 promotes follicular thyroid carcinoma progression via miRNAs sponge. Cell Death Dis 9: 372, 2018.

11. Teng H, Wang P, Xue Y, Liu X, Ma J, Cai H, Xi Z, Li Z and Liu Y: Role of HCP5-miR-139-RUNX1 feedback loop in regulating malignant behavior of glioma cells. Mol Ther 24: 1806-1822, 2016.

12. Wei X, Gu X, Ma M and Lou C: Long noncoding RNA HCP5 suppresses skin cutaneous melanoma development by regulating RARRES3 gene expression via sponging miR-12. Onco Targets Ther 12: 6323-6335, 2019.

13. Wang J and Wang GG: No easy way out for EZH2: Its pleiotropic, noncanonical effects on gene regulation and cellular function. Int J Mol Sci 21: 9501, 2020.

14. Xie Q, Wang H, Heilman ER, Walsh MG, Haseeb MA and Gupta R: Increased expression of enhancer of Zeste Homolog 2 (EZH2) differentiates squamous cell carcinoma from normal skin and actinic keratosis. Eur J Dermatol 24: 41-45, 2014.

15. Chen L, Jia J, Zang Y, Li J and Wan B: MicroRNA-101 regulates autophagy, proliferation and apoptosis via targeting EZH2 in laryngeal squamous cell carcinoma. Neoplasma 66: 507-515, 2019.

16. Liu Z, Yang L, Zhong C and Zhou L: EZH2 regulates H2B phosphorylation and elevates colon cancer cell autophagy. J Cell Physiol 235: 1494-1503, 2020.

17. Zuo S, Li X, Bao W and Li S: Pre-mRNA processing factor 3 enhances the progression of keratinocyte-derived cutaneous squamous cell carcinoma by regulating the JAK2/STAT3 pathway. Sci Rep 10: 8863, 2020.

18. Livak KJ and Schmittgen TD: Analysis of relative gene expression data using real-time quantitative PCR and the 2(-Delta Delta C(T)) method. Methods 25: 402-408, 2001.

19. Kopaladze RA: Methods for the euthanasia of experimental animals-the ethics, esthetics and personnel safety. Usp Fiziol Nauk 31: 79-90, 2000 (In Russian).

20. Zatroch KK, Knight CG, Reimer JN and Pang DS: Refinement of intraperitoneal injection of sodium pentobarbital for euthanasia in laboratory rats (Rattus norvegicus). BMC Vet Res 13: 60, 2017. 
21. Pan X, Chen Y, Shen Y and Tantai J: Knockdown of TRIM65 inhibits autophagy and cisplatin resistance in A549/DDP cells by regulating miR-138-5p/ATG7. Cell Death Dis 10: 429, 2019.

22. Zhao C, Ling X, Li X, Hou X and Zhao D: MicroRNA-138-5p inhibits cell migration, invasion and EMT in breast cancer by directly targeting RHBDD1. Breast Cancer 26: 817-825, 2019.

23. Zhou W, Zhang S, Li J, Li Z, Wang Y and Li X: IncRNA TINCR participates in ALA-PDT-induced apoptosis and autophagy in cutaneous squamous cell carcinoma. J Cell Biochem 120: 13893-13902, 2019.

24. Zhao M, Hu X, Xu Y, Wu C, Chen J, Ren Y, Kong L, Sun S, Zhang L, Jin R and Zhou X: Targeting of EZH2 inhibits epithelial-mesenchymal transition in head and neck squamous cell carcinoma via regulating the STAT3/VEGFR2 axis. Int J Oncol 55: 1165-1175, 2019.

25. Egolf S and Capell BC: LSD1: A viable therapeutic target in cutaneous squamous cell carcinoma? Expert Opin Ther Targets 24: 671-678, 2020.

26. Filoni A, Cicco G, Lospalluti L, Maglietta A, Foti C Annichiarico G, Resta L and Bonamonte D: Morphological and morphometric analysis of cutaneous squamous cell carcinoma in patients with recessive dystrophic epidermolysis bullosa: A retrospective study. J Eur Acad Dermatol Venereol 34: 1707-1714, 2020.

27. Hassan S, Purdie KJ, Wang J, Harwood CA, Proby CM, Pourreyron C, Mladkova N, Nagano A, Dhayade S, Athineos D, et al: A unique panel of patient-derived cutaneous squamous cell carcinoma cell lines provides a preclinical pathway for therapeutic testing. Int J Mol Sci 20: 3428, 2019.

28. Balasubramanian S, Adhikary G and Eckert RL: The Bmi-1 polycomb protein antagonizes the (-)-epigallocatechin-3-gallate-dependent suppression of skin cancer cell survival. Carcinogenesis 31: 496-503, 2010.

29. Zhuang Z, Xie N, Hu J, Yu P, Wang C, Hu X, Han X, Hou J, Huang $\mathrm{H}$ and Liu X: Interplay between $\Delta \mathrm{Np} 63$ and miR-138-5p regulates growth, metastasis and stemness of oral squamous cell carcinoma. Oncotarget 8: 21954-21973, 2017.

30. Yu Y, Shen HM, Fang DM, Meng QJ and Xin YH: LncRNA HCP5 promotes the development of cervical cancer by regulating MACC1 via suppression of microRNA-15a. Eur Rev Med Pharmacol Sci 22: 4812-4819, 2018.

31. Yun WK, Hu YM,Zhao CB, Yu DY and Tang JB: HCP5 promotes colon cancer development by activating AP1G1 via PI3K/AKT pathway. Eur Rev Med Pharmacol Sci 23: 2786-2793, 2019.

32. Han C, Sun B, Wang W, Cai W, Lou D, Sun Y and Zhao X: Overexpression of microtubule-associated protein-1 light chain 3 is associated with melanoma metastasis and vasculogenic mimicry. Tohoku J Exp Med 223: 243-251, 2011.

33. Mathew R, Karp CM, Beaudoin B, Vuong N, Chen G, Chen HY, Bray K, Reddy A, Bhanot G, Gelinas C, et al: Autophagy suppresses tumorigenesis through elimination of p62. Cell 137: 1062-1075, 2009.
34. Wright TJ, McKee C, Birch-Machin MA, Ellis R, Armstrong JL and Lovat PE: Increasing the therapeutic efficacy of docetaxel for cutaneous squamous cell carcinoma through the combined inhibition of phosphatidylinositol 3-kinase/AKT signalling and autophagy. Clin Exp Dermatol 38: 421-423, 2013.

35. Liu Y, Wang J, Dong L, Xia L, Zhu H, Li Z and Yu X: Long noncoding RNA HCP5 regulates pancreatic cancer gemcitabine (GEM) resistance by sponging Hsa-miR-214-3p to target HDGF. Onco Targets Ther 12: 8207-8216, 2019.

36. Manikandan M, Deva Magendhra Rao AK, Rajkumar KS, Rajaraman R and Munirajan AK: Altered levels of miR-21, miR-125b-2*, miR-138, miR-155, miR-184, and miR-205 in oral squamous cell carcinoma and association with clinicopathological characteristics. J Oral Pathol Med 44: 792-800, 2015.

37. Zheng S, Zhang X, Wang X and Li J: Downregulation of miR-138 predicts poor prognosis in patients with esophageal squamous cell carcinoma. Cancer Biomark 20: 49-54, 2017.

38. Tian S, Guo X, Yu C, Sun C and Jiang J: miR-138-5p suppresses autophagy in pancreatic cancer by targeting SIRT1. Oncotarget 8 : 11071-11082, 2017.

39. Liu X, Jiang L, Wang A, Yu J, Shi F and Zhou X: MicroRNA-138 suppresses invasion and promotes apoptosis in head and neck squamous cell carcinoma cell lines. Cancer Lett 286: 217-222, 2009.

40. Hernandez-Ruiz E, Toll A, Garcia-Diez I, Andrades E, Ferrandiz-Pulido C, Masferrer E, Yébenes M, Jaka A, Gimeno J, Gimeno R, et al: The Polycomb proteins RING1B and EZH2 repress the tumoral pro-inflammatory function in metastasizing primary cutaneous squamous cell carcinoma. Carcinogenesis 39: 503-513, 2018.

41. Chen H, Pan J, Zhang L, Chen L, Qi H, Zhong M, Shi X, Du J and Li Q: Downregulation of estrogen-related receptor alpha inhibits human cutaneous squamous cell carcinoma cell proliferation and migration by regulating EMT via fibronectin and STAT3 signaling pathways. Eur J Pharmacol 825: 133-142, 2018.

42. Bito T, Sumita N, Ashida M, Budiyanto A, Ueda M, Ichihashi M, Tokura $Y$ and Nishigori C: Inhibition of epidermal growth factor receptor and PI3K/Akt signaling suppresses cell proliferation and survival through regulation of Stat 3 activation in human cutaneous squamous cell carcinoma. J Skin Cancer 2011: 874571 , 2011.

43. Al-Dissi AN, Haines DM, Singh B and Kidney BA: Immunohistochemical expression of vascular endothelial growth factor and vascular endothelial growth factor receptor associated with tumor cell proliferation in canine cutaneous squamous cell carcinomas and trichoepitheliomas. Vet Pathol 44: 823-830, 2007. International (CC BY-NC-ND 4.0) License. 